\title{
ESCRITA ACADÊMICA DE PESQUISADORES APRENDIZES NO CIC-UFCG: CONSTATAÇÕES E REFLEXÕES
}

\author{
Roberta Andrade Meneses* \\ Williany Miranda da Silva**
}

\begin{abstract}
Resumo:A produção escrita de gêneros acadêmicos é um componente fundamental da formação em nível superior, não obstante, tal produção é comumente tomada por estudantes de graduação como complicada, difícil, e por que não dizer, incompreensível, já que, em geral, contrasta com as práticas de escrita advindas da escola. Desse modo, muitas das dimensões caracterizadoras da escrita acadêmica, em diferentes campos disciplinares, permanecem nebulosas durante toda a graduação, algo para o que contribui o caráter tácito das convenções que regem a escrita em domínio acadêmico. A partir deste quadro, o presente trabalho tem por objetivo oferecer maior visibilidade ao complexo cenário da aquisição de escrita especializada por graduandos inseridos em programas de pesquisa, os chamados pesquisadores aprendizes. Para tanto, analisamos abstracts, produzidos em língua portuguesa, publicados pelo CIC-UFCG (Congresso de Iniciação Científica da Universidade Federal de Campina Grande), na busca por marcas atestadoras da aquisição de escrita acadêmico-científica por parte dos referidos sujeitos. Os resultados indicam a existência de diferentes níveis de desenvolvimento de escrita, indiciados, principalmente, pela capacidade que os sujeitos apresentam de agenciar sua escrita, inscrevendo-se no discurso por meio da assunção à voz de pesquisador.
\end{abstract}

Palavras-chave: Letramento acadêmico-científico. Pesquisador aprendiz. Gênero abstract.

\begin{abstract}
The process of writing academic genres is a crucial component for higher education. However, this process is commonly considered by the students as complicated, difficult, and incomprehensible, for, generally, it contrasts with writing practices from school. Therefore, many characterizing dimensions of the academic writing, in different fields, still unclear during the whole graduation course. This unclearness contributes to a tacit character of the conventions behind the writing in academic environments. From this setting, this article aims to offer a wider visibility to the complex scenery of specialized writing acquisition by undergraduate students who participate on research programs as researcher apprentices. For this purpose, abstracts were analyzed. They were written in Portuguese and published by CIC-UFCG (Congress of Scientific Beginning of the Federal University of Campina Grande). We searched for evidences of the acquisition of academic-scientific writing by the individuals who wrote them. The results point an existence of different levels of writing development, indicted, mainly, by the capacity that the individuals presented in managing their writing, inserting themselves in the speech by the assumption to the researcher's voice.
\end{abstract}

Key words: Academic-scientific Literacy. Researcher apprentice. Abstract genre.

\section{Introdução}

A produção acadêmica é um nicho de estudos recorrentemente discutido nos dias atuais (BEZERRA, 2000; HENDGES, 2001; MOTTA-ROTH E HENDGES, 2010; FERREIRA, 2014; MENESES, 2013, 2015). A qualidade das produções, suas especificidades, as condições pragmáticas que as regem são algumas das questões sobre as quais diversos pesquisadores têm se debruçado, ora culminando em propostas cuja prevalência recai sobre a organização textual, ora sobre o contexto que reclama os textos.

\footnotetext{
* Professora da rede estadual de ensino da Paraíba, mestre em Linguagem e Ensino pelo Programa de PósGraduação em Letras (UFCG), especialista em Fundamentos da Educação (UEPB), graduada em LetrasPortuguês (UFCG) roberta.a.m@ hotmail.com.

** Professora da Unidade Acadêmica de Letras e do Programa de Pós-Graduação em Letras da Universidade Federal de Campina Grande. williany.miranda@gmail.com
} 
Em se tratando da esfera acadêmica, além da produção situada no âmbito pedagógico, isto é, no âmbito de textos relacionados ao processo de ensino/aprendizagem, há ainda os textos que materializam os chamados gêneros acadêmicos mais "profissionais" (BEZERRA, 2013), ligados fundamentalmente ao avanço do estado da arte nos mais diversos campos disciplinares.

É acerca desse segundo grupo que dedicamos nossa atenção no presente trabalho. Especificamente, enfocamos o gênero abstract ${ }^{1}$ produzido em língua portuguesa, que se enquadra no que genericamente chamamos resumo. No entanto, algumas particularidades o singularizam em relação a outras variedades de resumo, tais como, o abstract é produzido pelo próprio autor do texto-fonte, que pode ser um artigo, uma dissertação, uma tese; o abstract pode comportar-se ora como parte da composição de outros gêneros, situação em que surge compondo os próprios textos-fonte, ora como gênero autônomo, situação em que é enviado a periódicos especializados, seminários, colóquios, congressos, entre outros, para submissão de trabalhos à apreciação. No decorrer deste trabalho, a nomenclatura abstract estará sendo utilizada com esta última acepção.

Em nossa investigação, tratamos de abstracts produzidos por graduandos envolvidos com a produção de pesquisa, para os quais adotamos a denominação pesquisador aprendiz (Cf. PIRES, 2008). Trata-se de orientandos de iniciação cientifica, neste caso, vinculados ao Programa de bolsas de Iniciação Científica, doravante PIBIC, que produziram abstracts para a $13^{a}$ edição do Congresso de Iniciação Científica da Universidade Federal de Campina Grande, doravante CIC-UFCG.

O objetivo é identificar aspectos atestadores de letramento acadêmico-científico na produção escrita dos sujeitos, isto é, aspectos que indiciem padrões no uso da escrita alinhados às práticas de socialização acadêmico-científicas. Para tanto, orientamo-nos nas contribuições Swales (1990, 1994, 2009) acerca do estudo de gêneros; Motta-Roth e Hendges $(1996$; 2010) acerca do gênero abstract; Costa Val (1991) acerca do fator de informatividade; Mey, acerca do conceito de voz (2001); Beaufort (1998) acerca dos conhecimentos para escrita especializada; bem como Street (2010) acerca da concepção de letramento.

Dado nosso propósito, organizamos o trabalho em mais três seções, além desta introdução, a saber, Funcionamento do gênero abstract: a comunicação em pesquisas; Letramento acadêmico-científico: a voz do pesquisador; por fim, temos nossas conclusões, seguidas das referências utilizadas no trabalho.

\section{Funcionamento do gênero abstract: a comunicação em pesquisas}

Pensar a escrita acadêmica depreende compreender a academia enquanto um espaço de atuações diversificadas. Em se tratando do contexto de ensino superior no Brasil, essa diversificação, em termos gerais, pode ser alinhada aos dois propósitos básicos atribuídos a essa instância de ensino: a) busca sistemática por produção de saber, a pesquisa científica; b) busca por saber puro, pronto e dado, com vistas à profissionalização (ORTEGA Y GASSET, 1999).

Esse cenário de diversidade provoca desdobramentos para a natureza das práticas de letramento na academia, já que, conforme Street (2010), diferentes contextos - neste caso, os contextos de busca por produção de saber e de busca por saber pronto demandarão diferentes práticas de letramento. Desse modo, é de se esperar que as

\footnotetext{
${ }^{1} \mathrm{O}$ gênero que aqui tomamos por abstract, em consonância com Motta-Roth e Hendges (2010), pode receber outras denominações, tais quais, resumo, resumo de comunicação.
} 
práticas de letramento, isto é, os aspectos que segundo o autor nos permitem perceber padrões no uso da leitura e da escrita, variem também na academia.

Em se tratando do gênero abstract, estudos indicam sua inclusão entre os chamados gêneros de comunicação/divulgação científica, intimamente relacionados à prática do pesquisador e ao avanço do estado da arte nos diversos campos disciplinares (MENESES, 2015; MOTTA-HOTH e HENDGES, 2010; BEHLING, 2008).

Para Bhatia (1993), o gênero funciona como uma descrição capaz de sintetizar uma pesquisa completa, oferecendo ao leitor um conhecimento exato do TF. Quanto ao esperado para o gênero, o estudioso aponta que a organização do texto se dá em torno das seguintes questões: "1. O que o autor fez; 2. Como o autor o fez; 3. O que o autor encontrou; 4. O que o autor concluiu". Assim, ao organizar seu texto de modo a responder tais questões, o produtor aumenta as chances de que o efeito desejado com o resumo $^{2}$ seja alcançado.

Conforme podemos observar, a organização informacional do gênero resume aspectos tomados enquanto centrais a um trabalho de pesquisa, destacando-se essencialmente à delimitação do estudo, a metodologia utilizada, os resultados obtidos e a conclusão advinda destes. Coadunando-se a essa constatação, Motta-Roth e Hendges (2010), a partir de uma abordagem sociointeracionista de letramento científico, apresentam uma descrição esquemática da organização retórica do gênero abstract ${ }^{3}$, na qual atestam a aplicabilidade e adaptabilidade do chamado modelo CARS (Creat a Research Space) para a análise de gêneros. Considerado uma das mais significativas contribuições de John M. Swales para os estudos de análise de gêneros, o modelo CARS trata da análise do gênero a partir da depreensão de como o discurso se organiza em dois níveis hierárquicos de informação: os moves - estágios textuais e funcionais específicos de caráter mais abrangente; e os steps - estágios textuais de caráter menos abrangente.

O move ou movimento retórico é, então, uma fração do texto que realiza função comunicativa específica, constituindo, junto a outros movimentos, a totalidade da estrutura informacional do texto, permitindo que este seja reconhecido como exemplar de um determinado gênero (MOTTA-ROTH e HENDGES, 1998, p.127). Já os steps ou subfunções ( $C f$. MOTTA-ROTH e HENDGES, 2010) se subordinam aos movimentos, em alguns casos, constituindo-os.

A seguir, vejamos a descrição esquemática de organização retórica do gênero abstract proposta pelas autoras:

\footnotetext{
${ }^{2}$ Embora estejamos utilizando a denominação resumo, em respeito ao termo utilizado pelo autor citado (BHATIA, 1993), entenda-se que nos referimos à variedade de resumo que neste trabalho, em consonância com o proposto por Motta-Roth e Hendges (2010), estamos denominando de abstract.

${ }^{3}$ A descrição retoma dados de investigação anterior das autoras (MOTTA-ROTH e HENDGES, 1996), baseada na análise de 60 abstracts, metade em língua inglesa, metade em língua portuguesa, publicados em periódicos especializados.
} 
Figura 1- Descrição esquemática de abstracts. MOVIMENTO 1 SITUAR A PESQUISA

Subfunção 1A - Estabelecer interesse profissional no tópico ou

Subfunção 1B - Fazer generalizações no tópico e/ou

Subfunção 2A - Citar pesquisas prévias ou

Subfunção 2B - Estender pesquisas prévias ou

Subfunção 2C - Contra-argumentar pesquisas prévias ou

Subfunção 2D - Indicar lacunas em pesquisas prévias

MOVIMENTO 2 APRESENTAR A PESQUISA

Subfunção $1 \mathrm{~A}$ - Indicar as principais características ou

Subfunção 1B - Apresentar os principais objetivos e/ou

Sub-função 2 - Levantar hipóteses

\section{MOVIMENTO 3 DESCREVER A METODOLOGIA}

\section{MOVIMENTO 4 SUMARIZAR OS RESULTADOS}

\section{MOVIMENTO 5 DISCUTIR A PESQUISA}

Subfunção 1 - Elaborar conclusões e/ou

Subfunção 2 - Recomendar futuras aplicações

Fonte: Motta-Roth e Hendges (1996)

O estudo das autoras constatou, conforme se vê na figura 1, que os autores de abstracts, em sua maioria, realizam cinco movimentos retóricos para construção de seus textos: O movimento 1 - Situar a Pesquisa; O movimento 2 - Apresentar a Pesquisa; O movimento 3 -Descrever a metodologia; o movimento 4 -Sumarizar os Resultados e o movimento 5 - Discutir a Pesquisa; sendo recorrente, ainda, a construção de algumas subfunções nos movimentos 1,2 e 5 .

Ao considerarmos o CIC-UFCG, observamos que o evento disponibiliza normas e modelos para auxiliar a produção do gênero abstract. Na edição investigada neste estudo, o evento disponibilizou dois exemplares de abstracts tomados como modelos, produzidos por participantes do evento em edições anteriores, bem como um conjunto de normas, as quais podem vistas no quadro que segue:

Quadro 1- Normas para produção do Abstract no CIC-UFCG.

RESUMO: O texto deve iniciar-se na linha seguinte do item, ser claro, sucinto e, obrigatoriamente, explicar $\mathrm{o}(\mathrm{s})$ objetivo(s) pretendido(s) procurando justificar sua importância (sem incluir referências bibliográficas), os principais procedimentos $\underline{\text { adotados, }}$ os resultados mais expressivos e conclusões, contendo no máximo $\underline{14 \text { linhas. }}$

Abaixo devem aparecer as Palavras-chave (03 no máximo), procurando-se não repetir palavras do título, escritas em letras minúsculas.

Fonte: http://150.165.111.246/ sai/pesquisa2/index.php/todos-os-arquivos/category/14-modelosrelatorios

Ao observamos tais normas, percebemos que algumas relações podem ser estabelecidas com a descrição esquemática apresentada por Motta-Roth e Hendges (2010). Trata-se de algumas afinidades, perceptíveis por meio da presença de movimentos retóricos comuns tanto ao modelo descritivo quanto às normas, conforme se vê na figura a seguir, que indica a estrutura retórica preconizada pelas normas do congresso para a produção de abstracts: 
Figura 2- Organização retórica preconizada pelas orientações normativas do CIC-UFCG para a produção de Abstract.

\begin{tabular}{|c|c|}
\hline & do CIC-UFCG para a produção de Abstract. \\
Movimento 2 \\
\hline $1-$ & APRESENTAR O(S) OBJETIVO(S) DOTRABALHO \\
\hline 2- & JUSTIFICATICAR REALIZAÇÃO DO TRABALHO \\
\hline & Movimento 3 \\
\hline & DESCREVER A METODOLOGIA \\
\hline Movimento 4 \\
\hline SUMARIZAR OS RESULTADOS \\
Movimento 5 \\
\hline ELABORAR CONCLUSÕES
\end{tabular}

Fonte: Elaborado pela autora (2017)

A figura 2 evidencia que quatro dos cinco movimentos retóricos encontrados na descrição esquemática do gênero abstract por Motta-Roth e Hendges (1998) surgem também nas normas da $13^{\mathrm{a}}$ edição do CIC-UFCG. Encontrar afinidades entre modelo descritivo e prescritivo indica um panorama de certo consenso quanto ao funcionamento esperado para o gênero. Desse modo, vale considerar que a comparação entre perspectivas de descrição e de normatização, conforme nosso estudo indicia, pode constituir um método relevante à verificação de consensos e dissensos no que tange ao funcionamento de gêneros. No caso em questão, percebe-se certa estabilização no que concerne ao gênero enquanto síntese dos aspectos centrais de uma pesquisa, notabilizando-se que sua organização retórica aponta para movimentos retóricos ligados, genericamente, à contextualização da pesquisa - Apresentar a pesquisa, Situar a pesquisa, Descrever metodologia -, bem como para contribuição que a pesquisa, dado o próprio caráter do trabalho científico, deve trazer para a área do conhecimento em questão- Sumarizar resultados, Discutir a pesquisa.

No entanto, vários estudos indicam que a frequência com que os movimentos retóricos apresentados aparecem constituindo abstracts é irregular, notadamente quando se comparam diferentes áreas (MOTTA-ROTH e HENDGES, 1998; MENESES, 2013; MENESES, 2015), de modo que, por uma lado, os movimentos mais coadunados à contextualização mostram-se mais pacificamente realizáveis e, por outro, os movimentos que efetivam contribuições para a área do conhecimento mostram-se menos pacificamente realizáveis.

Nesse sentido, o movimento 2 - Apresentar a pesquisa tem se mostrado como o movimento mais recorrentemente realizado em abstracts de diferentes áreas, notadamente por meio da Subfunção - Apresentar os principais objetivos, conforme nos demonstra estudo realizado com um corpus de 45 abstracts, divididos igualmente entre as áreas de Humanas, Saúde e Exatas (MENESES, 2015).

Tal fato nos indica que a proposição de objetivo(s) é condição consensual em se tratando de relatar pesquisas em qualquer dessas áreas. Por outro lado, ainda conforme esse estudo, o movimento 5 - Discutir a pesquisa é o menos recorrente nas três áreas, realidade corroborada por resultados de outros trabalhos enfocando o abstract (MOTTA-ROTH e HENDGES, 1998; MENESES, 2013). Este último dado pode se explicar pelo fato de que ao discutir a pesquisa, por meio de elaboração de conclusões ou indicações de aplicações futuras, o autor precisa lançar uma interpretação acerca de seus resultados e não somente apresentá-los, o que depreende um processo cognitivo mais complexo.

$\mathrm{Na}$ próxima seção, vejamos como tais constatações dialogam com as expectativas do CIC-UFCG e de seus autores para o gênero abstract. 


\section{Letramento acadêmico-científico: a voz do pesquisador}

Ao investigarmos abstracts produzidos por graduandos bolsistas de programa de iniciação científica, para submissão de trabalhos de pesquisa ao CIC-UFCG, compreendemos que tais sujeitos assumem papeis por meio de seus textos, ou, conforme Mey (2001), assumem vozes, isto é, expressam a ocupação de um determinado lugar na sociedade. No caso em questão, as implicações decorrentes dos aspectos pragmáticos observados - ser bolsista de iniciação científica, produtor de abstract, que submete trabalho de pesquisa a congresso - nos habilita a compreensão de que esses autores devam assumir a voz de pesquisadores, ainda que aprendizes.

Assim sendo, podemos avaliar o letramento acadêmico-científico a partir do reconhecimento coletivo de padrões de produção e recepção de determinado gênero deste domínio, padrões que, no caso ora investigado, são orquestrados pela voz - ou papel social - do pesquisador. Portanto, as normas do evento para a produção do abstract, bem como as descrições esquemáticas do gênero, que apontam para como autores o têm produzido, constituem evidências de reconhecimento coletivo de um padrão, senão estável, ao menos discernível.

Considerando que tratamos de pesquisadores aprendizes, é esperado que diferentes níveis de letramento acadêmico-científico se notabilizem, a depender de como os abstracts desses produtores atendem às condições de reconhecimento de funcionamento do gênero, que conforme já salientado, diz respeito à ação de divulgar/comunicar pesquisas. Considerando ainda o papel social do pesquisador, ligado à produção do conhecimento, compreendemos que entre os movimentos retóricos apontados enquanto recorrentes para constituição do gênero, os movimentos 4Sumarizar resultados e 5-Discutir a pesquisa, este último notadamente por meio da elaboração de conclusões, são sobretudo, mas não exclusivamente, responsáveis pela instauração da voz do pesquisador, já que por meio desses movimentos o autor pode desvencilhar-se da mera reprodução das fontes, contribuindo para o conhecimento na área, de modo a dotar seu texto de informatividade.

Ao tratar do nível de informatividade ${ }^{4}$ em redações vestibulares, Costa Val (1991) toma esse fator de textualidade a partir das noções de suficiência de dados e previsibilidade. Ancorados por esta perspectiva, tomaremos a informatividade a partir da identificação de indícios da voz do pesquisador nos abstracts analisados, isto é, as constatações, teses e asserções que constituam acréscimo de conhecimento à determinada área de estudos, extrapolando a mera reprodução de conhecimento dado.

Além da informatividade, capaz de trazer dados novos à experiência do interlocutor, também consideraremos em nossa análise os conhecimentos que, em domínio acadêmico, podem determinar a condição de inserção e participação de indivíduos nesta comunidade discursiva ${ }^{5}$, trata-se de conhecimentos que se imbricam para a produção de escrita especializada, isto é, a escrita que materializa os gêneros típicos da comunidade discursiva acadêmica. Segundo Beaufort (1998 apud SWALLES, 2009), cinco são esses conhecimentos: (1) conhecimento do assunto, (2) conhecimento retórico, (3) conhecimento do processo de escrita, (4) conhecimento do gênero e (5) conhecimento da comunidade discursiva.

\footnotetext{
${ }^{4}$ Fatores de textualidade são as características que fazem com um texto seja tomado como texto, são eles: coesão, coerência, intencionalidade, aceitabilidade, situacionalidade, informatividade e intertextualidade (BEAUGRANDE e DRESSLER, 1983 apud COSTA VAL, 1991).

5 Comunidade discursiva acadêmica, conforme Swales (1990), pode ser entendida um grupo de indivíduos partilhando objetivos públicos comuns (há também os interesses particulares - implícitos ou explícitos).
} 
A seguir, vejamos a análise do abstract 1:

Abstract 1

História Ambiental e Atividade Baleeira na Paraíba: Organização e estudo compreensivo da documentação disponível em Lucena/PB. (1978-1989)

\section{RESUMO}

A década de 1970 é marcada pela ascensão dos temas ligados a conscientização ambiental em todo mundo. Neste período diversas entidades não governamentais surgiram, tendo a defesa do meio ambiente como foco principal. Neste contexto, surge em 1978 a Associação Paraibana Amigos da Natureza APAN, que irá empreender esforços para acabar com a atividade baleeira em Lucena, litoral Norte do Estado da Paraíba. Atividade estabelecida desde 1912, com a instalação da Copesbra - Companhia de Pesca Norte do Brasil, servindo de base econômica para o referido município/Estado, onde cerca de Três mil habitantes dependiam direta ou indiretamente da atividade. Com isso, até a proibição da caça a partir da Lei federal 7.683 no ano de 1987, todo um debate entre ecologistas, parlamentares e a população é noticiado pelos jornais da época de 1970 e 1980.

Palavras Chave: Meio Ambiente, Atividade baleeira, Lei Federal 7.683/1987

Exemplo 4 - Abstract produzido pelo sujeito 1 Fonte: http://www.pibic.ufcg.edu.br/anais/2013/

Acessado em $12 \backslash 12 \backslash 2014$

Conforme podemos constatar, o abstract 1, publicado nos anais do evento em sua $13^{\mathrm{a}}$ edição, evidencia um sujeito com adequada performance no que tange ao processo de escrita, havendo articulação das ideias por meio de mecanismos coesivos, o que se atesta, por exemplo, pelo recurso da progressão referencial ${ }^{6}$, conforme primeira e segunda linhas do texto, em que a expressão "A década de 70 [...]" é retomada por "Neste período [...]"; ou ainda na linha cinco, em que a progressão ocorre por meio de reiteração: “[...] a atividade baleeira em Lucena [...] (L4) / Atividade estabelecida [...] (L5). Tal quadro nos indica um sujeito ciente do processo de escrita, com clara preocupação em oferecer ao texto um encadeamento lógico de progressão. Além disso, o texto também nos indica um autor que tem conhecimento do assunto de que trata, demonstrando que o sujeito 1 realizou, de fato, um levantamento de fontes sobre a história ambiental e atividade baleeira na Paraíba.

No entanto, do ponto de vista do conhecimento retórico, do conhecimento do gênero e do conhecimento da comunidade discursiva acadêmica, o texto evidencia que o sujeito 1 encontra-se distante de uma postura consciente acerca das implicações pragmáticas que envolvem sua participação no CIC-UFCG, visto ignorar convenções importantes para aceitabilidade de seu texto em círculos científicos.

No que tange à estrutura retórica, há ausência de construção dos movimentos de apresentação de um objetivo, justificativa, descrição de métodos, apresentação dos resultados, elaboração de conclusões - aspectos importantes não apenas porque são cobrados pelo CIC-UFCG, mas porque se consolidaram enquanto meios eficientes de construção do discurso científico ${ }^{7}$.

O texto, portanto, não apresenta, conforme se espera para o gênero, a sistematização do trabalho de pesquisa, apresentando, na verdade, uma síntese de suas fontes, descrevendo-nos aquilo que elas trazem: "A década de 1970 é marcada pela

\footnotetext{
${ }^{6}$ A progressão referencial, conforme Koch e Elias (2009, p. 132), diz do fenômeno de referentes que, uma vez dados, são retomados mais adiante no texto ou servem de base para introdução de novos referentes.

${ }^{7}$ A consolidação desses movimentos como meios de construção do discurso científico pode ser atestada pela recorrência com que surgem em gêneros do domínio científico - relatórios, artigos, projetos de pesquisa, entre outros.
} 
ascensão dos temas ligados a conscientização ambiental em todo mundo [...] Neste contexto, surge em 1978 a Associação Paraibana Amigos da Natureza [...].” Trata-se, sem dúvida, de um tipo de resumo, mas não de um com características de abstract. Em outras palavras, o sujeito 1 promove nada mais que uma apresentação do conhecimento dado, presente nas fontes, na documentação disponível.

Tal perspectiva parece muito mais alinhada ao tipo de resumo encontrado em âmbito pedagógico, no qual o resumo funciona enquanto uma avaliação de leitura. Segundo Silva (2012), este tipo de resumo, também conhecido como resumo escolar ou acadêmico, funciona como uma atividade de avaliação de leitura na qual o aluno expõe as ideias centrais de um texto-fonte, demonstrando ser capaz de ler, compreender e registrar linguisticamente tal compreensão.

Como se percebe, trata-se de um tipo de resumo no qual os traços do autor tendem a ser apagados, já que seu propósito é sintetizar conhecimento dado, resumindo o texto-fonte. Do ponto de vista do conteúdo informacional, esse tipo de resumo se caracteriza pelo critério de suficiência de dados - conforme os objetivos de leitura - mas não necessariamente pelo critério de imprevisibilidade, já que o conteúdo exposto existe na íntegra na forma do texto-fonte.

Considerando que o texto 1 procura se inserir no âmbito científico e não no âmbito pedagógico, seu nível de informatividade pode ser considerado baixo, posto que não se percebe a instauração da voz do pesquisador na escrita, isto é, não há produção de teses e asserções que evidenciem sua posição diante dos dados, o que se tem, na verdade, é um texto que não extrapola o que é presumível a partir do acesso às fontes, visto não haver a formalização de resultados ou conclusões que apontem para o trabalho de elaboração de conhecimentos - ratificações, refutações, descobertas - para a área.

Vejamos a análise do abstract 2, que segue:

Modelo - abstract disponibilizado pelo CIC-UFCG (2013)

\section{INFLUÊNCIA DA VIOLÊNCIA URBANA NAS REDEFINIÇÕES DO USO DOS ESPAÇOS PÚBLICOS DE CAMPINA GRANDE - PB. \\ MOVIMENTO 1}

A preocupação com o espacos público têm ganhado um espaço cada vez mais significativo no debate sobre a dinâmica urbana ao se caracterizarem como um dos ambientes mais vulneráveis aos problemas sociais. Um dos fatores que definem novas perspectivas destes sujeitos é o fenômeno da insegurança,

\section{MOVIMENTO 4}

conforme observado nos resultados obtidos na pesquisa de mapeamento da violência urbana em Campina Grande realizada no período 2010/2011, na qual se identificou uma alta incidência de crimes no centro e bairros próximos, os quais concentram alto fluxo de pessoas e áreas destinadas ao lazer, mas que tem sido noticiada e distanciada do convívio dos campinenses.

\section{MOVIMENTO 2}

Diante desse quadro, o trabalho ora apresentado, buscou analisar a influência da violência urbana no uso e criação de identidades com estes espaços públicos.

\section{MOVIMENTO 3}

Para isto, foram selecionados cinco espaços tidos como estratégicos para a ambiência urbana na cidade: Parque do povo, Feira Central; Praça da Bandeira; Parque da Criança e Feira da Prata. A pesquisa utilizou como aporte metodológico as técnicas da pesquisa qualiquantitativa: Aplicação de questionários em espaços públicos; obtenção de Dados Oficiais; e entrevistas com habitantes.

\section{PROPOSTA DE MOVIMENTO 5}

A guisa de CONCLUSÃO percebeu-se a interferência da violência e o medo no uso principalmente da Praça da Bandeira localizada no Centro da cidade.

Palavras-chave: ambiência urbana, insegurança, medo.

Fonte: http://150.165.111.246/ sai/pesquisa2/index.php/todos-os-arquivos/category/14modelos-relatorios 
O abstract 2, ora enfocado, foi um dos exemplares selecionados pelo CICUFCG para servir de modelo para produção do gênero. Ao analisá-lo, percebemos que este se encontra organizado em quatro movimentos retóricos, embora o autor intente, conforme nos demonstra o uso de marcadores metadiscursivos" ("conclusão", "percebeu-se"), realizar um quinto movimento, o qual, conforme veremos adiante, acaba por não se realizar efetivamente.

O primeiro deles é o movimento 1 - Contextualizar/introduzir a pesquisa, no qual o autor nos apresenta algumas generalizações acerca do tema - "A preocupação com o espaços públicos têm ganhado um espaço cada vez mais significativo no debate sobre a dinâmica urbana [...]". Neste caso, percebemos que os resultados do trabalho são apresentados no início do texto, amalgamando-se ao movimento 1 - "nos resultados obtidos na pesquisa [...] se identificou uma alta incidência de crimes [...]" - seguidos da continuação do movimento 2, agora por meio da apresentação do objetivo do trabalho, - "buscou analisar a influência da violência urbana no uso e criação de identidades com estes espaços públicos [...]". Este fato pode indicar certa dificuldade do autor em discriminar e ordenar logicamente as informações, hipótese corroborada pela indistinção feita entre Resultados e Conclusões, conforme veremos mais adiante.

O trecho seguinte é referente ao movimento 3- Descrever a Metodologia, no qual o autor indica os procedimentos, o tipo de pesquisa e seus instrumentos - "foram selecionados cinco espaços [...]"; "A pesquisa utilizou como aporte metodológico as técnicas da pesquisa qualiquantitativa"; "[...] questionários em espaços públicos; obtenção de Dados Oficiais; e entrevistas com habitantes".

O último trecho do texto, embora seja anunciado como o movimento de Discutir a Pesquisa, por meio da elaboração de conclusões - "A guisa de conclusão [...]" - é, na verdade, a apresentação de mais um resultado do estudo - "[...] percebeu-se a interferência da violência e o medo no uso principalmente da Praça da Bandeira [...]". Essa realidade nos leva a duas hipóteses: a) $\mathrm{O}$ autor demonstra dificuldade em diferenciar a natureza desses dois movimentos; b) A tentativa de construir o movimento 5 pode estar ligada à preocupação do autor em enquadrar-se às normas para produção do abstract disponibilizadas pelo evento.

$\mathrm{Na}$ análise do abstract, chama a atenção ainda, a ocorrência de problemas de construção que dificultam a coerência do texto e demonstram a inabilidade do autor em reconhecer os referentes para a adequada articulação dos períodos. Além da ausência total de referente, tal qual ocorre na terceira linha do movimento 1 , no qual o autor apresenta uma informação nova como se fosse informação dada, já conhecida pelo leitor - "[...] Um dos fatores que definem novas perspectivas destes sujeitos [...]" - quais sujeitos?

Em suma, do ponto de vista do conhecimento do processo de escrita, o texto acima pode ser considerado um texto problemático, com presença de incoerências e, inclusive, desvios da norma culta (verbo ter acentuado com sujeito no singular; ausência de crase na expressão "A guisa"; ausência de concordância em trechos como "A preocupação com o espaços públicos"). No entanto, do ponto de vista dos conhecimentos retórico, do gênero e da comunidade discursiva, o texto oferece condições de ser reconhecido como exemplar de abstract. Tal realidade pode ser atestada pela organização retórica apresentada, que se coaduna, ainda que parcialmente, tanto às normas do evento, quanto ao que se espera do gênero, conforme nos indicam perspectivas descritivas, já referidas. Além disso, a adoção de uma linguagem marcada por aspectos tradicionalmente esperados para a produção de gêneros acadêmicos-

\footnotetext{
${ }^{8}$ Marcadores metadiscursivos são pistas lexicais que caracterizam os tipos de informações que compõem o texto (MOTTA-ROTH e HENDGES, 2010).
} 
científicos $^{9}$, como a opção pela neutralidade, evidenciadas por uso da $3^{\text {a }}$ pessoa e construções passivas indicam a ação de certo conhecimento da comunidade discursiva em questão.

Assim sendo, ao ser escolhido como modelo pelo evento, ainda que apresentando questões merecedoras de reformulação, o texto 2 evidencia que para os avaliadores do CIC-UFCG o conhecimento do processo de escrita foi considerado menos relevante que os conhecimentos retórico, do gênero e da comunidade discursiva acadêmica. Vale observar, todavia, que a análise realizada pelo evento parece ser marcada por um rastreamento de superfície, já que a mera indicação de um movimento, conforme vimos, nem sempre significou a efetiva construção do movimento retórico anunciado, caso atestado no movimento de Discutir a pesquisa por meio da elaboração de conclusões, cujo anúncio não significou efetiva construção do movimento.

Por fim, no que tange ao nível de informatividade, o abstract 2 traz dados e constatações, notadamente por meio do movimento de sumarizar resultados: "[...] se identificou uma alta incidência de crimes no centro e bairros próximos[...] percebeu-se a interferência da violência e o medo no uso principalmente da Praça da Bandeira [...]"; tal realidade atesta a ação do pesquisador sobre seu objeto de estudo, resultando em contribuições, acréscimos para o conhecimento na área. Assim, embora apresente aspectos problemáticos sem dúvida relevantes, o texto 2 consegue inserir-se no âmbito acadêmico-científico, já que além de indiciar a ação dos conhecimentos retórico, do gênero e da comunidade discursiva acadêmica, encontra-se dotado de um nível de informatividade capaz de legar conhecimento à área.

\section{Conclusões}

A análise da escrita de pesquisadores aprendizes, materializada no gênero acadêmico-científico abstract pôde nos revelar alguns aspectos relacionados ao processo de aquisição dessa escrita especializada. Tais aspectos foram identificados a partir dos critérios de nível de informatividade, bem como pela ação dos conhecimentos requeridos para produção de escrita especializada, indicando que os abstracts analisados apontam para diferentes níveis de desenvolvimento de escrita acadêmico-científica por parte de seus autores. No abstract 1, o conhecimento do processo de escrita não constituiu condição suficiente para que o texto atendesse as condições de funcionamento do gênero conforme indicado tanto nas expectativas relacionadas à descrição quanto à normatização do gênero. Em outras palavras, ratificamos o que já nos indica Beaufort (1998 apud SWALLES, 2009) de que é necessária a ação integrada de diferentes conhecimentos para produção de texto especializado, de modo que o conhecimento do processo de escrita não constitui condição suficiente para estabelecimento de letramento acadêmico-científico.

No que tange ao abstract 2, pudemos constatar a ação dos conhecimentos retórico, do gênero, da comunidade discursiva acadêmica, muito embora o texto tenha justamente evidenciado falhas significativas no que tange ao conhecimento do processo de escrita. Além desse aspecto, o abstract 2 traz um dado interessante ao demonstrar que os marcadores metadiscursivos podem funcionar, por vezes, não para introduzir a construção efetiva de um movimento retórico, mas antes para simulá-lo.

\footnotetext{
${ }^{9}$ Reconheçamos que ainda que a busca pela neutralidade seja recorrentemente tomada como ideal aos textos acadêmico-científicos, tal critério não constitui aspecto balizador absoluto de cientificidade, mas antes um aspecto da tradição. No mais, vale destacar que as condições de efetivação da neutralidade é tema atualmente controverso em diversos círculos científicos.
} 
Por fim, no que se refere ao nível de informatividade de ambos os textos, é possível perceber que somente o abstract 2 apresenta nível significativo, considerando o âmbito no qual os dois textos se inserem, o científico. Nesse sentido, o nível de informatividade se liga à capacidade que o autor tem de extrapolar a mera reprodução das fontes, inscrevendo-se no discurso por meio da apresentação de dados, formulação de asserções, teses que possam representar contribuição para a área de estudos na qual atua.

\section{Referências}

BEHLING, J. Resumos de comunicação e o agenciamento da escrita científica. Dissertação - UEC - Campinas, SP, 2008.

BEZERRA, B. G. Letramentos acadêmicos na perspectiva dos gêneros textuais. Fórum Linguístico, Florianópolis, v. 9, n. 4, p. 247-258, out./dez. 2012. Disponível em: <http://dx.doi.org/10.5007/1984-8412.2012v9n4p247>. Acesso em: 22 mar. 2013.

BHATIA, Vijay K. Analyzing genre: language use in professional settings. London: Longman, 1993.

COSTA VAL, M. G. Redação e textualidade. São Paulo: Martins Fontes, 1999.

FERREIRA, C; A. Desenvolvimento da escrita na academia: investigação longitudinal do percurso de licenciandos em Letras. Dissertação de Mestrado. Unidade Acadêmica de Letras, Universidade Federal de Campina Grande, Campina Grande, PB, 2014.

MEY, J. L. As vozes da sociedade: Seminários de pragmática. Campinas, SP: Mercado da Letras, 2001.

MENESES, R. A. Produção de Abstracts no VIII Congresso de Iniciação Científica da UFCG: O que é requerido e o que é demonstrado? Monografia (Trabalho de Conclusão de Curso) - Unidade Acadêmica de Letras, Universidade Federal de Campina Grande, Campina Grande, PB, 2013.

MENESES, R. A. A escrita acadêmica do pesquisador aprendiz: abstracts em eventos científicos. Dissertação de Mestrado. Unidade Acadêmica de Letras, Universidade Federal de Campina Grande, Campina Grande, PB, 2015.

MOTTA-ROTH, D.; HENDGES, G. R. Uma análise transdiciplinar do gênero Abstract. In: Intercâmbio. Vol. 7: 1998, p. 125-134.

MOTTA-ROTH, D.; HENDGES, G. R. Produção textual na universidade. São Paulo: Parábola, 2010, p. 151 - 162.

ORTEGA Y GASSET, J. Missão da universidade. Rio de Janeiro: EdUERJ, 1999.

PIRES, R. C. M. A formação inicial do professor pesquisador universitário no Programa Institucional de Bolsas de Iniciação Científica - PIBIC/CNPq e a prática 
profissional de seus egressos: Um estudo de caso na Universidade do Estado da Bahia. Tese - UFRS - Porto Alegre, RS, 2008

SILVA, E. M. Professora como é que se faz? Campina Grande, PB: Bagagem, 2012.

STREET, B. Os novos estudos sobre o letramento: histórico e perspectivas. In.: MARINHO. M.;CARVALHO, G. T. (Orgs.) Cultura escrita e letramento. Belo Horizonte: Editora UFMG, 2010.

SWALES, J.; FEAK, C. Academic Writing for Graduate Students, Ann Arbor, the university of Michigan Press, 1994.

SWALES, J. M. Sobre modelos de análise do discurso. In: BIASI-RODRIGUES, Bernardete; ARAÚJO, Júlio César; SOUSA, Socorro Cláudia Tavares de (Orgs.). Gêneros textuais e comunidades discursivas: um diálogo com John Swales. Belo Horizonte: Autêntica Editora, 2009. (Coleção Leitura, Escrita e Oralidade). 